Research Article

\title{
Exact Number of Positive Solutions for a Class of Two-Point Boundary Value Problems
}

\author{
Yanmin Niu and Baoqiang Yan \\ School of Mathematical Sciences, Shandong Normal University, Jinan 250014, China \\ Correspondence should be addressed to Baoqiang Yan; yanbaoqiang666@gmail.com
}

Received 25 September 2013; Accepted 4 November 2013

Academic Editor: Yansheng Liu

Copyright ( 2013 Y. Niu and B. Yan. This is an open access article distributed under the Creative Commons Attribution License, which permits unrestricted use, distribution, and reproduction in any medium, provided the original work is properly cited.

This paper considers the existence of positive solutions of the boundary value problems $v^{\prime \prime}+\lambda\left(v^{r}+v^{p}-v^{q}\right)=0$ and $v(-1)=v(1)=0$, where $p>r>q>-1$ and $\lambda$ is a positive parameter. Using a time-map approach, we obtain the exact number of positive solutions in different cases.

\section{Introduction and Main Results}

The study of multiplicity results to boundary value problem

$$
\begin{gathered}
v^{\prime \prime}+\lambda f(v)=0, \quad t \in(a, b), \\
v(a)=v(b)=0,
\end{gathered}
$$

where $\lambda>0$ is a positive parameter, is very interesting because of its applications. As we know, when $f(v)=v^{p}(x)+$ $v^{q}(x)$, the boundary value problem

$$
\begin{gathered}
-v^{\prime \prime}(x)=\mu v^{p}(x)+v^{q}(x), \quad a \leq x \leq b, \\
v(x)>0, \quad x \in(a, b), \\
v(a)=v(b)=0,
\end{gathered}
$$

where $0<q<1<p$ and $k \geq 0$ are fixed given numbers and $\mu>0$ is a parameter, comes from the elliptic problem

$$
\begin{gathered}
-\Delta u=\lambda u^{q}+u^{p}, \quad x \in \Omega, \\
u>0, \quad x \in \Omega, \\
u=0, \quad x \in \partial \Omega,
\end{gathered}
$$

with $0<q<1<p$, which was raised by Ambrosetti et al. in [1].

Under different assumptions on $f$, there are many results for the above problems and elliptic equations (see [2-8]).

In $[9,10]$, Liu considered the case of $f(v)=v^{p}+(1 / \lambda) v^{q}$ and $f(v)=v^{p}+v^{q}+k v$ and gave the exact number of solutions and many interesting properties of the solutions.
Cheng [11] investigated the following two-point boundary value problem:

$$
\begin{gathered}
-y^{\prime \prime}=\lambda\left(y^{p}-y^{q}\right), \quad t \in(-1,1), \\
y(-1)=y(1)=0,
\end{gathered}
$$

where $\lambda>0$ is a positive parameter and $p>q>-1$ and got the exact number of positive solutions.

Now, in this paper we consider the more general case

$$
\begin{gathered}
-v^{\prime \prime}=\lambda\left(v^{r}+v^{p}-v^{q}\right), \quad t \in(-1,1), \\
v(-1)=v(1)=0,
\end{gathered}
$$

where $\lambda>0$ is a positive parameter, $p>r>q>-1$, and $(1 /(r+1))+(1 /(p+1))-(1 /(q+1))<0$.

Define $\beta$, where $\beta$ satisfies

$$
\frac{\beta^{r}}{r+1}+\frac{\beta^{p}}{p+1}-\frac{\beta^{q}}{q+1}=0 .
$$

For $p>r>q$ and $-1<q<1$, let $\lambda_{1}$ be given by

$$
\begin{aligned}
\lambda_{1} & \frac{r+1}{2} \beta^{1-r} \\
& \times\left(\int_{0}^{1} \frac{d t}{\sqrt{t^{q+1}\left[\left(1-t^{r-q}\right)+((r+1) /(p+1)) \beta^{p-r}\left(1-t^{p-q}\right)\right]}}\right)^{2} .
\end{aligned}
$$

The main results of this paper are as follows. 
Theorem 1. If $p>r>q \geq 1$, (5) has exactly one positive solution for any $\lambda>0$.

Theorem 2. If $p>r \geq 1>q>-1$, (5) has exactly one positive solution for $\lambda \in\left(0, \lambda_{1}\right]$ and none for $\lambda>\lambda_{1}$.

Theorem 3. If $p>1>r>q \geq 0$, (5) has exactly one positive solution for $\lambda \in\left(0, \lambda_{1}\right]$ and none for $\lambda>\lambda_{1}$.

Theorem 4. If $(1 / 3) \geq p>r>q \geq 0$, (5) has exactly one positive solution for $\lambda \in\left(\lambda_{1},+\infty\right)$ and none for $\lambda<\lambda_{1}$.

Theorem 5. Assume that $1>p>r>0>q>-1$. Define

$$
\begin{aligned}
\theta(r, p, q) & =\int_{0}^{1} \frac{1-r}{\left[t^{q+1}\left(1-m_{1} \beta^{r-q} t^{r-q}-m_{2} \beta^{p-q} t^{p-q}\right)\right]^{1 / 2}} d t \\
& -(r-q) \int_{0}^{1} \frac{1-t^{q+1}}{\left[t^{q+1}\left(1-m_{1} \beta^{r-q} t^{r-q}-m_{2} \beta^{p-q} t p^{p-q}\right)\right]^{3 / 2}} d t \\
& -\frac{(p-r)(q+1)}{p+1} \beta^{p-q} \\
& \times \int_{0}^{1} \frac{1-t^{p+1}}{\left[t^{q+1}\left(1-m_{1} \beta^{r-q} t^{r-q}-m_{2} \beta^{p-q} t^{p-q}\right)\right]^{3 / 2}} d t,
\end{aligned}
$$

where $m_{1}=(q+1) /(r+1)$ and $m_{2}=(q+1) /(p+1)$. One has the following.

(1) If $\theta(r, p, q) \geq 0$, (5) has exactly one positive solution for $\lambda \in\left(\lambda_{1},+\infty\right)$ and none for $\lambda \in\left(0, \lambda_{1}\right)$.

(2) If $\theta(r, p, q)<0$, there exists $\lambda_{0} \in\left(0, \lambda_{1}\right)$ such that (5) has exactly two positive solutions for $\lambda \in\left(\lambda_{0}, \lambda_{1}\right]$, exactly one for $\lambda \in\left(\lambda_{1},+\infty\right)$ or $\lambda=\lambda_{0}$, and none for $\lambda \in\left(0, \lambda_{0}\right)$.

\section{The Proofs of Theorems 1-4}

We assume throughout this section that $p>r>q>-1$ and

$$
\frac{1}{r+1}+\frac{1}{p+1}-\frac{1}{q+1}<0 .
$$

Denote that $E=(\beta,+\infty)$, where $\beta$ is given by (6). For $a \geq \beta$ and $t \in(0,1)$, let

$$
P(a, t)=\int_{a t}^{a}\left(z^{r}+z^{p}\right) d z, \quad Q(a, t)=\int_{a t}^{a} z^{q} d z .
$$

Define a function $F: E \rightarrow(0,+\infty)$ as

$$
F(a)=\int_{0}^{a}\left[2 \int_{v}^{a}\left(z^{r}+z^{p}-z^{q}\right) d z\right]^{-1 / 2} d v \quad \text { for } a \in E .
$$

It is clear that $\int_{0}^{\beta}\left(z^{r}+z^{p}-z^{q}\right) d z=0$. Now we claim that $a^{r}+$ $a^{p}-a^{q}>0$ for $a \geq \beta$ and $\int_{0}^{a}\left[2 \int_{v}^{a}\left(z^{r}+z^{p}-z^{q}\right) d z\right]^{-1 / 2} d v<\infty$ if and only if $a \in E$.
Let $g_{1}(z)=z^{p}$ and $g_{2}(z)=z^{r}-z^{q}$; then $f(z)=$ $g_{1}(z)+g_{2}(z)$. Immediately, we get that $g_{2}^{\prime}(x)=r z^{r-1}-q z^{q-1}=$ $z^{q-1}\left(r z^{r-q}-q\right)$. In order to judge the sign of $g_{2}^{\prime}(x)$, we just judge the sign of $T(z)=r z^{r-q}-q$. Since $T(0)=-q, T(1)=$ $r-q$, and $T^{\prime}(z)=r(r-q) z^{r-q-1}$, we can get the following two results.

(1) For $r>q>0$, the function $g_{2}(z)$ has a stable point $z_{0}=(q / r)^{1 /(r-q)}$. When $z \in\left(0, z_{0}\right)$, we have $g_{2}^{\prime}(z)<0$, and when $z \in\left(z_{0},+\infty\right)$, we have $g_{2}^{\prime}(z)>0$.

(2) For $r>0>q$, we have $g_{2}^{\prime}(z)>0$ on $z \in(0,+\infty)$. Combining $g_{1}^{\prime}(x)=p z^{p-1}$ and $g_{1}^{\prime \prime}(x)=p(p-1) z^{p-2}$ with the above two results, we obtain the monotony of $f(z)$.

(3) For $p \geq 1>r>q>0$, the function $f(z)$ has a stable point $z_{*}$ on $(0,1)$. When $z \in\left(0, z_{*}\right)$, we have $f^{\prime}(z)<0$ and when $z \in\left(0, z_{*}\right)$, we have $f^{\prime}(z)>0$.

(4) For $1>p>r>q>0$, we have that $f^{\prime}(z)>0$ on $(0,+\infty)$.

(5) For $p>r>0>q$ or $p>0>r>q$, we have that $f^{\prime}(z)>0$ on $(0,+\infty)$.

Then from $\int_{0}^{\beta}\left(z^{r}+z^{p}-z^{q}\right) d z=0$, we infer that $a^{r}+a^{p}-a^{q}>0$ for $a \geq \beta$.

We consider the integration $\int_{0}^{a}\left[2 \int_{v}^{a}\left(z^{r}+z^{p}-\right.\right.$ $\left.\left.z^{q}\right) d z\right]^{-1 / 2} d v$. It is clear that $v=a$ is a flaw point. Since $\int_{0}^{a}\left[2 \int_{v}^{a}\left(z^{r}+z^{p}-z^{q}\right) d z\right]^{-1 / 2} d v<\int_{0}^{a}\left[2 \int_{v}^{a}\left(z^{p}-z^{q}\right) d z\right]^{-1 / 2} d v$ on $E$, we consider the integration $\int_{0}^{a}\left[2 \int_{v}^{a}\left(z^{p}-z^{q}\right) d z\right]^{-1 / 2} d v$.

Using Lagrange theorem, we obtain that

$$
\begin{aligned}
& \int_{0}^{a}\left[2 \int_{v}^{a}\left(z^{p}-z^{q}\right) d z\right]^{-1 / 2} d v \\
& =\int_{0}^{a}\left[2\left(\frac{a^{p+1}}{p+1}-\frac{v^{p+1}}{p+1}\right)\right. \\
& \left.\quad-2\left(\frac{a^{q+1}}{q+1}-\frac{v^{q+1}}{q+1}\right)\right]^{-1 / 2} d v \\
& =\int_{0}^{a}\left\{2\left[v+\theta_{1}(a-v)\right]^{p}(a-v)\right. \\
& =\int_{0}^{a}[2(a-v)]^{-1 / 2}\left\{\left[v+\theta_{1}(a-v)\right]^{p}\right. \\
& \left.\quad-\left[v+\theta_{2}(a-v)\right]^{q}\right\}^{-1 / 2} d v
\end{aligned}
$$

where $\theta_{1}, \theta_{2} \in(0,1)$ are constants.

The following Lemma 6 is listed to show that to study the number of positive solutions of (5) is equivalent to study the shape of the map $F(a)$ on $E$. Lemmas 7-9 show some properties of $F(a)$ on $E$. 
Lemma 6. Let $u(a, t)$ be the unique solution of the problem

$$
\begin{gathered}
0 \leq u(t) \leq a, \\
\int_{\mathcal{u}(t)}^{a}\left[2 \int_{v}^{a}\left(z^{r}+z^{p}-z^{q}\right) d z\right]^{-1 / 2} d v=F(a) t, \\
t \in[0,1],
\end{gathered}
$$

where $a \in E$. One has the following.

(1) If $\lambda>0$ and $v$ is a positive solution of (5), $v(0) \in E$, $F(v(0))=\sqrt{\lambda}$, and $v(t)=u(v(0),|t|)$ for $t \in[-1,1]$.

(2) If $a \in E$ and $F(a)=\sqrt{\lambda}, v(t)=u(v(0),|t|)$ is a positive solution of (5) with $v(0)=a$.

Proof. (1) Assume that $\lambda>0$ and $v$ is a positive solution of (5). Let $\tau \in[-1,1]$ satisfy $v(\tau)=\max _{t \in[-1,1]} v(t)=a$. It follows from $v^{\prime}(\tau)=0$ that

$$
\begin{aligned}
{\left[v^{\prime}(s)\right]^{2} } & =\int_{\tau}^{s} 2 v^{\prime \prime}(\eta) v^{\prime}(\eta) d \eta \\
& =2 \lambda \int_{v(s)}^{a}\left(z^{r}+z^{p}-z^{q}\right) d z, \quad s \in(-1,1) .
\end{aligned}
$$

This implies that $a \geq \beta$ and $v^{\prime}(s) \neq 0$ if $v(s)<a$. And combine $v^{\prime \prime}(\tau)=-\lambda\left(a^{r}+a^{p}-a^{q}\right)<0$ to obtain

$$
\begin{array}{ll}
v^{\prime}(s)>0 & \text { for } s \in(-1, \tau), \\
v^{\prime}(s)<0 & \text { for } s \in(\tau, 1) .
\end{array}
$$

Then, we have that

$$
\begin{aligned}
& v^{\prime}(s)\left[2 \int_{v(s)}^{a}\left(z^{r}+z^{p}-z^{q}\right) d z\right]^{-1 / 2}=\sqrt{\lambda}, \quad s \in(-1, \tau), \\
& -v^{\prime}(s)\left[2 \int_{v(s)}^{a}\left(z^{r}+z^{p}-z^{q}\right) d z\right]^{-1 / 2}=\sqrt{\lambda}, \quad s \in(\tau, 1) .
\end{aligned}
$$

It follows that

$$
\begin{gathered}
\int_{v(t)}^{a}\left[2 \int_{v}^{a}\left(z^{r}+z^{p}-z^{q}\right) d z\right]^{-1 / 2} d v=(\tau-t) \sqrt{\lambda}, \\
t \in[-1, \tau], \\
\int_{v(t)}^{a}\left[2 \int_{v}^{a}\left(z^{r}+z^{p}-z^{q}\right) d z\right]^{-1 / 2} d v=(t-\tau) \sqrt{\lambda}, \\
t \in[\tau, 1], \\
(1-\tau) \sqrt{\lambda}=\int_{0}^{a}\left[2 \int_{v}^{a}\left(z^{r}+z^{p}-z^{q}\right) d z\right]^{-1 / 2} d v \\
=(\tau+1) \sqrt{\lambda} .
\end{gathered}
$$

From (19) and $a \geq \beta$ we have that $\tau=0$. With (17) and (18) we obtain the result (1) of this theorem.

(2) Since $u(a, 0)=a>0$ and $u(a, t)$ is a positive solution of the boundary value problem

$$
\begin{gathered}
u^{\prime \prime}+[F(a)]^{2}\left(u^{r}+u^{p}-u^{q}\right)=0, \quad t \in(0,1), \\
u^{\prime}(0)=0, \quad u(1)=0,
\end{gathered}
$$

we have that $v(t)=u(a,|t|)$ is a positive solution of (5).

Lemma 7. $F$ is differentiable on $(\beta, \infty)$, and

$$
\begin{array}{ll}
F(a)=\frac{1}{4 \sqrt{2}} \int_{0}^{1} H_{0}(a, t) d t, & a>\beta, \\
F^{\prime}(a)=\frac{1}{4 \sqrt{2}} \int_{0}^{1} H_{1}(a, t) d t, & a>\beta,
\end{array}
$$

where

$$
\begin{gathered}
H_{0}(a, t)=4 a[P(a, t)-Q(a, t)]^{-1 / 2}, \\
H_{1}(a, t)=2[P(a, t)-Q(a, t)]^{-3 / 2} \\
\times\left[\frac{1-r}{r+1} a^{r+1}\left(1-t^{r+1}\right)+\frac{1-p}{p+1} a^{p+1}\left(1-t^{p+1}\right)\right. \\
\left.\quad-\frac{1-q}{q+1} a^{q+1}\left(1-t^{q+1}\right)\right] .
\end{gathered}
$$

Proof. Equation (21) can be obtained by (11), immediately. From

$$
\begin{aligned}
\frac{\partial P(a, t)}{\partial a}= & \frac{\partial}{\partial a} \\
& \times\left[\frac{1-r}{r+1} a^{r+1}\left(1-t^{r+1}\right)+\frac{1-p}{p+1} a^{p+1}\left(1-t^{p+1}\right)\right] \\
= & a^{r}\left(1-t^{r+1}\right)+a^{p}\left(1-t^{p+1}\right), \\
\frac{\partial Q(a, t)}{\partial a} & =\frac{\partial}{\partial a}\left[\frac{1}{q+1} a^{q+1}\left(1-t^{q+1}\right)\right]=a^{q}\left(1-t^{q+1}\right),
\end{aligned}
$$

we have that

$$
\begin{aligned}
\frac{\partial H_{0}(a, t)}{\partial a}= & 2[P(a, t)-Q(a, t)]^{-3 / 2} \\
\times & {\left[\frac{1-r}{r+1} a^{r+1}\left(1-t^{r+1}\right)+\frac{1-p}{p+1} a^{p+1}\left(1-t^{p+1}\right)\right.} \\
& \left.\quad-\frac{1-q}{q+1} a^{q+1}\left(1-t^{q+1}\right)\right] \\
= & H_{1}(a, t) .
\end{aligned}
$$

It follows from (21) that $F$ is differentiable on $(\beta, \infty)$ and (22) is true. 
Lemma 8. Consider the following:

$$
\lim _{a \rightarrow \beta} F(a)= \begin{cases}+\infty, & q \geq 1, \\ \sqrt{\lambda_{1}}, & q<1,\end{cases}
$$

where $\lambda_{1}$ is given by (7).

Proof. From

$$
\lim _{a \rightarrow \beta} \int_{\beta}^{a} \frac{d v}{\sqrt{2 \int_{v}^{a}\left(z^{r}+z^{p}-z^{q}\right) d z}}=0
$$

and the Lebesgue theorem, we have that

$$
\begin{aligned}
\lim _{a \rightarrow \beta} F(a) & =\lim _{a \rightarrow \beta} \int_{0}^{\beta} \frac{d v}{\sqrt{2 \int_{v}^{a}\left(z^{r}+z^{p}-z^{q}\right) d z}} \\
& =\int_{0}^{\beta} \frac{d v}{\sqrt{2 \int_{v}^{\beta}\left(z^{r}+z^{p}-z^{q}\right) d z}} .
\end{aligned}
$$

On the other hand, from $\int_{0}^{\beta}\left(z^{r}+z^{p}-z^{q}\right) d z=0$, we can obtain that

$$
\begin{aligned}
& \int_{0}^{\beta} \frac{d v}{\sqrt{2 \int_{v}^{\beta}\left(z^{r}+z^{p}-z^{q}\right) d z}} \\
& =\int_{0}^{\beta} \frac{d v}{\sqrt{2 \int_{0}^{v}\left(z^{q}-z^{r}-z^{p}\right) d z}} \\
& =\int_{0}^{1}(\beta d t) \\
& =\int_{0}^{1}\left(\sqrt{2\left[\frac{(\beta t)^{q+1}}{q+1}-\frac{(\beta t)^{r+1}}{r+1}-\frac{(\beta t)^{p+1}}{p+1}\right]}\right)^{-1} \\
& \times\left(2 \left[\frac{\beta^{r+1}}{r+1} t^{q+1}+\frac{\beta^{p+1}}{p+1} t^{q+1}\right.\right. \\
& \left.\left.=\sqrt{\frac{r+1}{2} \beta^{(1-r) / 2}} \frac{(\beta t)^{r+1}}{r+1}-\frac{(\beta t)^{p+1}}{p+1}\right]\right)^{-1 / 2} \\
& \quad \times \sqrt{t^{q+1}\left[\left(1-t^{r-q}\right)+((r+1) /(p+1)) \beta^{p-r}\left(1-t^{p-q}\right)\right]}
\end{aligned}
$$

This completes the proof of Lemma 8 .

Lemma 9. Consider the following:

$$
\lim _{a \rightarrow+\infty} F(a)= \begin{cases}0, & p>1, r \geq 1 \text { or } p>1, r<1 \\ \frac{\pi}{2 \sqrt{2}}, & p=1, r=1 \\ +\infty, & p<1, r<1\end{cases}
$$

Proof. From

$$
\begin{aligned}
& \lim _{a \rightarrow+\infty} \frac{Q(a, t)}{P(a, t)} \\
& =\lim _{a \rightarrow+\infty} \frac{a^{q+1}\left(1-t^{q+1}\right) /(q+1)}{a^{r+1}\left(1-t^{r+1}\right) /(r+1)+a^{p+1}\left(1-t^{p+1}\right) /(p+1)} \\
& =\lim _{a \rightarrow+\infty}\left(\left(1-t^{q+1}\right)(r+1)(p+1)\right) \\
& \quad \quad \times\left((q+1)(p+1) a^{r-q}\left(1-t^{r+1}\right)\right. \\
& \left.\quad+(q+1)(r+1) a^{p-q}\left(1-t^{p+1}\right)\right)^{-1} \\
& =0,
\end{aligned}
$$

we have that

$$
\begin{aligned}
& \lim _{a \rightarrow+\infty} \frac{a}{\sqrt{P(a, t)-Q(a, t)}} \\
& =\lim _{a \rightarrow+\infty} \frac{a}{\sqrt{P(a, t)}} \\
& =\lim _{a \rightarrow+\infty} \frac{1}{\sqrt{(1 /(r+1)) a^{r-1}\left(1-t^{r+1}\right)+(1 /(p+1)) a^{p-1}\left(1-t^{p+1}\right)}} .
\end{aligned}
$$

From Lemma 7 and the Lebesgue theorem, we can obtain the results of this lemma.

In the following section, we give the proofs of Theorems $1-5$. For convenience, we denote that

$$
\begin{aligned}
I(a, t)= & \frac{1-r}{r+1} a^{r+1}\left(1-t^{r+1}\right)+\frac{1-p}{p+1} a^{p+1}\left(1-t^{p+1}\right) \\
& -\frac{1-q}{q+1} a^{q+1}\left(1-t^{q+1}\right) .
\end{aligned}
$$

Hence from Lemma 7, we have that

$$
F^{\prime}(a)=\frac{1}{4 \sqrt{2}} \int_{0}^{1} 2(P-Q)^{-3 / 2} I(a, t) d t, \quad \text { for } a>\beta .
$$

Proof of Theorem 1. From $p>r>q \geq 1$, we obtain that

$$
\begin{aligned}
I(a, t)< & \frac{1-r}{r+1} a^{r+1}\left(1-t^{r+1}\right)+\frac{1-p}{p+1} a^{p+1}\left(1-t^{p+1}\right) \\
& -\frac{1-q}{q+1} a^{p+1}\left(1-t^{p+1}\right) \\
= & \frac{1-r}{r+1} a^{r+1}\left(1-t^{r+1}\right)+\frac{2(q-p)}{(p+1)(q+1)} a^{p+1} \\
& \times\left(1-t^{p+1}\right)<0,
\end{aligned}
$$

for $a>\beta, t \in(0,1)$. Thus $F^{\prime}(a)<0$ for $a>\beta$. By Lemmas 6 , 8 , and 9 , we have the results of this theorem.

Proof of Theorem 2. From $p>r \geq 1>q>-1$, (33), and (34), we have that $F^{\prime}(a)<0$ for $a>\beta$. It follows from Lemmas 6 , 8 , and 9 that the results of Theorem 2 hold. 
Proof of Theorem 3. Conditions $p>1>r>q \geq 0$ and $(1 /(r+$ $1))+(1 /(p+1))-(1 /(q+1))<0$ imply that $r p-1>q(r+p+2)>$ 0 . With (33), we obtain that

$$
\begin{aligned}
I(a, t)< & \frac{1-r}{r+1} a^{p+1}\left(1-t^{p+1}\right)+\frac{1-p}{p+1} a^{p+1}\left(1-t^{p+1}\right) \\
& -\frac{1-q}{q+1} a^{q+1}\left(1-t^{q+1}\right) \\
< & \frac{2(1-r p)}{(p+1)(r+1)} a^{p+1}\left(1-t^{p+1}\right)-\frac{1-q}{q+1} a^{q+1} \\
& \times\left(1-t^{q+1}\right)<0,
\end{aligned}
$$

for $a>\beta, t \in(0,1)$, which means that $F^{\prime}(a)<0$ for $a>$ $\beta$. Thus by Lemmas 6,8 , and 9 , we have the results of this theorem.

Proof of Theorem 4. From (1/3) $\geq p>r>q \geq 0$ and (33), we have that

$$
\begin{aligned}
I(a, t)> & \frac{1-r}{r+1} a^{q+1}\left(1-t^{q+1}\right)+\frac{1-p}{p+1} a^{q+1}\left(1-t^{q+1}\right) \\
& -\frac{1-q}{q+1} a^{q+1}\left(1-t^{q+1}\right) \\
> & \left(2 \frac{1-p}{p+1}-\frac{1-q}{q+1}\right) a^{q+1}\left(1-t^{q+1}\right) \\
= & \frac{q(3-p)+(1-3 p)}{(p+1)(q+1)} a^{q+1}\left(1-t^{q+1}\right)>0
\end{aligned}
$$

for $a>\beta, t \in(0,1)$. Hence $F^{\prime}(a)>0$ for $a>\beta$. By Lemmas 6,8 , and 9 , we have the results of this theorem.

\section{The Proof of Theorem 5}

In this section we always assume that $1>p>r>0>q>-1$. Denote that

$$
\begin{array}{r}
S(a, t)=\frac{P(a, t)}{Q(a, t)}, \quad a>\beta, t \in(0,1), \\
h_{1}(s)=(s-1)(s-n), \quad s \in(-\infty, \infty), \\
h_{2}(s)=-3(s-m)(s-n)+2(s-1)(s-l), \\
s \in(-\infty, \infty),
\end{array}
$$

where $P(a, t), Q(a, t)$, and $\beta$ are given by (10), (6), and

$$
\begin{array}{r}
m=m(t)=\frac{q+1}{r+1}-\frac{(p-r)(q+1)}{(p+1)(r+1)} a^{p-q} \frac{1-t^{p+1}}{1-t^{q+1}}, \\
a>\beta, \quad t \in(0,1), \\
n=n(t)=\frac{1-q}{1-r}+\frac{(p-r)(q+1)}{(p+1)(1-r)} a^{p-q} \frac{1-t^{p+1}}{1-t^{q+1}}, \\
a>\beta, \quad t \in(0,1), \\
l=l(t)=\frac{1-q^{2}}{1-r^{2}}+\frac{\left(p^{2}-r^{2}\right)(q+1)}{(p+1)\left(1-r^{2}\right)} a^{p-q} \frac{1-t^{p+1}}{1-t^{q+1}}, \\
a>\beta, \quad t \in(0,1) .
\end{array}
$$

Remark 10. From $1>p>r>0 \geq q>-1$, (6), and (41), it is obvious that $m<1, n>1$, and $l<n$.

Lemma 11. For $a>\beta$ and $t \in(0,1)$,

$$
\frac{q+1}{r+1} a^{r-q}+\frac{q+1}{p+1} a^{p-q}<\frac{P(a, t)}{Q(a, t)}<a^{r-q}+a^{p-q} .
$$

Proof. Let

$$
\begin{aligned}
K(t)= & \frac{a^{r}}{r+1}\left[(r-q) t^{r+q+1}+(q+1) t^{q}-(r+1) t^{r}\right] \\
& +\frac{a^{p}}{p+1}\left[(p-q) t^{p+q+1}+(q+1) t^{q}-(p+1) t^{p}\right] .
\end{aligned}
$$

Condition $1>p>r>0>q>-1$ implies that

$$
\begin{aligned}
& K^{\prime}(t)=\frac{a^{r}}{r+1}\left[(r-q)(r+q+1) t^{r+q}\right. \\
& \left.\quad+(q+1) q t^{q-1}-(r+1) r t^{r-1}\right] \\
& +\frac{a^{p}}{p+1}\left[(p-q)(p+q+1) t^{p+q}\right. \\
& \left.\quad+(q+1) q t^{q-1}-(p+1) p t^{p-1}\right] \\
& <\frac{a^{r}}{r+1}[(r-q)(r+q+1) \\
& \quad+(q+1) q-(r+1) r] t^{r+q} \\
& +\frac{a^{p}}{p+1}[(p-q)(p+q+1)+(q+1) q \\
& \quad-(p+1) p] t^{p+q}=0, \quad t \in(0,1) .
\end{aligned}
$$

With $K(1)=0$ and (44), we have that $K(t)>0$ for $t \in(0,1)$. It follows that

$$
\frac{\partial}{\partial t} \frac{P(a, t)}{Q(a, t)}=\frac{q+1}{\left(1-t^{q+1}\right)^{2}} a^{-q} K(t)>0, \quad t \in(0,1)
$$

Combining

$$
\begin{gathered}
\lim _{t \rightarrow 0} \frac{P(a, t)}{Q(a, t)}=\frac{q+1}{r+1} a^{r-q}+\frac{q+1}{p+1} a^{p-q}, \\
\lim _{t \rightarrow 1} \frac{P(a, t)}{Q(a, t)}=a^{r-q}+a^{p-q}
\end{gathered}
$$

we have the results of this lemma.

Lemma 12. For $1>p>r>0>q>-1$,

$$
\lim _{a \rightarrow \beta} F^{\prime}(a)=\frac{1}{2 \sqrt{2}}\left(\frac{q+1}{\beta^{q+1}}\right)^{1 / 2} \theta(r, p, q) .
$$


Proof. From Lemma 7 we have that

$$
\begin{aligned}
2 \sqrt{2} F^{\prime}(a) & \int_{0}^{1}\left(\left(\frac{1-r}{r+1} a^{r+1}\left(1-t^{r+1}\right)+\frac{1-p}{p+1} a^{p+1}\left(1-t^{p+1}\right)\right.\right. \\
& \left.\quad-\frac{1-q}{q+1} a^{q+1}\left(1-t^{q+1}\right)\right) \\
& \left.\times\left([P(a, t)-Q(a, t)]^{3 / 2}\right)^{-1}\right) d t \\
= & \int_{0}^{1} \frac{1-r}{[P(a, t)-Q(a, t)]^{1 / 2}} d t \\
& -\frac{r-q}{q+1} a^{q+1} \int_{0}^{1} \frac{1-t^{q+1}}{[P(a, t)-Q(a, t)]^{3 / 2}} d t \\
& -\frac{p-r}{p+1} a^{p+1} \int_{0}^{1} \frac{1-t^{p+1}}{[P(a, t)-Q(a, t)]^{3 / 2}} d t .
\end{aligned}
$$

It follows from Lebesgue theorem that

$$
\begin{aligned}
& \lim _{a \rightarrow \beta} \int_{0}^{1} \frac{1-r}{[P(a, t)-Q(a, t)]^{1 / 2}} d t \\
&= \int_{0}^{1} \frac{1-r}{[P(\beta, t)-Q(\beta, t)]^{1 / 2}} d t \\
& \lim _{a \rightarrow \beta} \frac{r-q}{q+1} a^{q+1} \int_{0}^{1} \frac{1-t^{q+1}}{[P(a, t)-Q(a, t)]^{3 / 2}} d t \\
&+\lim _{a \rightarrow \beta} \frac{p-r}{p+1} a^{p+1} \int_{0}^{1} \frac{1-t^{p+1}}{[P(a, t)-Q(a, t)]^{3 / 2}} d t \\
&=\frac{r-q}{q+1} \beta^{q+1} \int_{0}^{1} \frac{1-t^{q+1}}{[P(\beta, t)-Q(\beta, t)]^{3 / 2}} d t \\
&+\frac{p-r}{p+1} \beta^{p+1} \int_{0}^{1} \frac{1-t^{p+1}}{[P(\beta, t)-Q(\beta, t)]^{3 / 2}} d t .
\end{aligned}
$$

Finally, $\int_{0}^{\beta}\left(z^{r}+z^{p}-z^{q}\right) d z=0$ implies that

$$
\begin{aligned}
P(\beta, t) & -Q(\beta, t) \\
= & \int_{0}^{\beta t}\left(z^{q}-z^{r}-z^{p}\right) d z \\
= & \frac{(\beta t)^{q+1}}{q+1}\left(1-m_{1} \beta^{r-q} t^{r-q}-m_{2} \beta^{p-q} t^{p-q}\right) .
\end{aligned}
$$

Combing (49) and (50), we complete the proof of this lemma.

Lemma 13. $F(a)$ has continuous derivatives up to second order on $(\beta, \infty)$ as follows:

$$
\begin{aligned}
& F^{\prime}(a)=\frac{1-r}{2 \sqrt{2}} \int_{0}^{1} G(a, t) h_{1}(S(a, t)) d t, \quad a>\beta, \\
& F^{\prime \prime}(a)=\frac{1-r^{2}}{4 a \sqrt{2}} \int_{0}^{1} G(a, t) h_{2}(S(a, t)) d t, \quad a>\beta,
\end{aligned}
$$

where $G(a, t)=[S(a, t)-1]^{-5 / 2}[Q(a, t)]^{-1 / 2}$.
Proof. Equation (51) can be obtained by

$$
\begin{aligned}
H_{1}(a, t)= & 2[P(a, t)-Q(a, t)]^{-3 / 2} \\
\times & {[(1-r) P(a, t)-(1-q) Q(a, t)} \\
& \left.-\frac{p-r}{p+1} a^{p+1}\left(1-t^{p+1}\right)\right] \\
= & 2\left[\frac{P(a, t)}{Q(a, t)}-1\right]^{-5 / 2}[Q(a, t)]^{-1 / 2} \\
& \times(1-r)\left[\frac{P(a, t)}{Q(a, t)}-\frac{1-q}{1-r}-\frac{(p-r)(q+1)}{(p+1)(1-r)}\right. \\
\times & \left.\times a^{p-q} \frac{1-t^{p+1}}{1-t^{q+1}}\right]\left[\frac{P(a, t)}{Q(a, t)}-1\right] \\
= & 2(1-r) G(a, t) h_{1}(S(a, t))
\end{aligned}
$$

and Lemma 7, immediately. From (24) we have that

$$
\begin{aligned}
& a \frac{\partial H_{1}(a, t)}{\partial a}=-3(P-Q)^{-5 / 2} \\
& \times[(r+1) P-(q+1) Q \\
& \left.+\frac{p-r}{p+1} a^{p+1}\left(1-t^{p+1}\right)\right] \\
& \times\left[(1-r) P-(1-q) Q-\frac{p-r}{p+1} a^{p+1}\right. \\
& \left.\times\left(1-t^{p+1}\right)\right]+2(P-Q)^{-3 / 2} \\
& \times\left[\left(1-r^{2}\right) P-\left(1-q^{2}\right) Q\right. \\
& \left.-\frac{p^{2}-r^{2}}{p+1} a^{p+1}\left(1-t^{p+1}\right)\right] \\
& =\left(\frac{P}{Q-1}\right)^{-3 / 2}\left(1-r^{2}\right) \\
& \times\left\{-3\left[P-\frac{q+1}{r+1} Q+\frac{(p-r)}{(p+1)(r+1)}\right.\right. \\
& \left.\times a^{p+1}\left(1-t^{p+1}\right)\right] \\
& \times\left[P-\frac{1-q}{1-r} Q-\frac{(p-r)}{(p+1)(1-r)} a^{p+1}\right. \\
& \left.\times\left(1-t^{p+1}\right)\right]+2(P-Q)
\end{aligned}
$$




$$
\begin{gathered}
\times\left[P-\frac{1-q^{2}}{1-r^{2}} Q-\frac{\left(p^{2}-r^{2}\right)}{(p+1)\left(1-r^{2}\right)}\right. \\
\left.\left.\times a^{p+1}\left(1-t^{p+1}\right)\right]\right\} \\
=\left(1-r^{2}\right) G(a, t) h_{2}(S(a, t)),
\end{gathered}
$$

and so (52) holds.

Lemma 14. There exist $\delta(r, p, q)>0$ and $\eta(r, p, q)>0$ such that

$$
\begin{aligned}
F^{\prime \prime}(a) & +\delta(r, p, q) F^{\prime}(a) \frac{1+r}{2 a} \\
> & \eta(r, p, q)\left(a^{r-q}+a^{p-q}\right)^{-5 / 2} a^{-(3+q) / 2} \quad \text { for } a>\beta .
\end{aligned}
$$

Proof. From (41) and $1>p>r>0>q>-1$, we have that

$$
\begin{aligned}
\min _{s \in[1, n]} h_{2}(s) & =\min \left\{h_{2}(1), h_{2}(n)\right\} \\
& =(n-1) \min \{3(1-m), 2(n-l)\} \\
& \geq 2(n-1) \min \{(1-m),(n-l)\} \\
& >2(n-1) \min \left\{\frac{r-q}{r+1}, \frac{(1-q)(r-q)}{1-r^{2}}\right\} \\
& =2(n-1) \frac{r-q}{r+1} .
\end{aligned}
$$

And since $h_{1}((1+n) / 2)=-\left((n-1)^{2} / 4\right)<0$, let $\delta(r, p, q)>0$ satisfy

$$
\min _{s \in[1, n]}\left[h_{2}(s)+\delta(r, p, q) h_{1}(s)\right]=2(n-1) \frac{r-q}{r+1} .
$$

Set

$$
h(s)=h_{2}(s)+\delta(r, p, q) h_{1}(s), \quad s \in(-\infty, \infty) .
$$

From $h(s)=(\delta-1) s^{2}+(3 m+3 n-2 l-2-\delta-\delta n) s-3 m n+2 l+\delta n$, we have that $\delta(r, p, q)-1>0$ and

$$
h(s) \geq \min _{s \in[1, n]} h(s)=2(n-1) \frac{r-q}{r+1}, \quad s \in(-\infty, \infty) .
$$

On the other hand, from (10), (38), and Lemma 11, we have that

$$
\begin{gathered}
0<Q(a, t)<\frac{1}{q+1} a^{q+1}, \\
0<\frac{q+1}{a^{q+1}} \int_{0}^{a}\left(z^{r}+z^{p}-z^{q}\right) d z \\
=\frac{q+1}{r+1} a^{r-q}+\frac{q+1}{p+1} a^{p-q}-1 \\
<S(a, t)-1<S(a, t)<a^{r-q}+a^{p-q},
\end{gathered}
$$

for $a>\beta, t \in(0,1)$.
This means that

$$
\begin{aligned}
G(a, t) & =[S(a, t)-1]^{-5 / 2}[Q(a, t)]^{-1 / 2} \\
& >\left(a^{r-q}+a^{p-q}\right)^{-5 / 2} a^{-(1+q) / 2} \sqrt{1+q},
\end{aligned}
$$

for $a>\beta, t \in(0,1)$. It follows from Lemma 13 and (58) that

$$
\begin{aligned}
F^{\prime \prime}(a) & +\delta(r, p, q) F^{\prime}(a) \frac{1+r}{2 a} \\
& =\frac{1-r^{2}}{4 a \sqrt{2}} \int_{0}^{1} G(a, t) h(S(a, t)) d t .
\end{aligned}
$$

Now, from (59)-(62) we have (55), where

$$
\eta(r, p, q)=\frac{(1-r)(r-q) \sqrt{1+q}}{2 \sqrt{2}} \int_{0}^{1}[n(t)-1] d t .
$$

Lemma 15. If $\theta(r, p, q) \geq 0$, then $F^{\prime}(a)>0$ for $a>\beta$. If $\theta(r, p, q)<0$, then there exists $a^{*}>\beta$, such that $F^{\prime}(a)<0$ for $a \in\left(\beta, a^{*}\right)$ and $F^{\prime}(a)>0$ for $a>a^{*}$.

Proof. It follows from Lemma 14 that if

$$
F^{\prime}(a)=0, \quad \text { then } F^{\prime \prime}(a)>0,
$$

and then

$$
F^{\prime}(a) \text { has at most one zero in }(\beta, \infty) \text {. }
$$

By (40), (42), and (51) we can obtain that

$$
F^{\prime}(a)>0 \text { for } \frac{P(a, t)}{\mathrm{Q}(a, t)}>n(t) .
$$

With (10) and (41), (66) implies that

$$
F^{\prime}(a)>0 \text { for } \frac{q+1}{r+1} a^{r}+\left(\frac{q+1}{p+1}-\frac{p-r}{1-r}\right) a^{p}>\frac{1-q}{1-r} a^{q} .
$$

If $\theta(r, p, q) \neq 0$, then from Lemma 12 and (64)-(67) we have the results of this lemma, immediately. If $\theta(r, p, q)=0$, then by Lemmas 12 and 14 we have that $F^{\prime \prime}(a)>0$ for $a$ near $\beta$, and so $F^{\prime}(a)>0$ for $a$ near $\beta$. Thus, it follows from (64)-(67) that $F^{\prime}(a)>0$ for $a>\beta$.

Proof of Theorem 5. From Lemmas 6, 8, 9, and 15, we can obtain the results of Theorem 5 .

\section{References}

[1] A. Ambrosetti, H. Brezis, and G. Cerami, "Combined effects of concave and convex nonlinearities in some elliptic problems," Journal of Functional Analysis, vol. 122, no. 2, pp. 519-543, 1994.

[2] P. Korman, Y. Li, and T. Ouyang, "Exact multiplicity results for boundary value problems with nonlinearities generalising cubic," Proceedings of the Royal Society of Edinburgh A, vol. 126, no. 3, pp. 599-616, 1996. 
[3] P. Korman and T. Ouyang, "Exact multiplicity results for two classes of boundary value problems," Differential and Integral Equations, vol. 6, no. 6, pp. 1507-1517, 1993.

[4] P. Korman and T. Ouyang, "Multiplicity results for two classes of boundary-value problems," SIAM Journal on Mathematical Analysis, vol. 26, no. 1, pp. 180-189, 1995.

[5] T. Laetsch, "The number of solutions of a nonlinear two point boundary value problem," Indiana University Mathematics Journal, vol. 20, pp. 1-13, 1970.

[6] J. Smoller and A. Wasserman, "Global bifurcation of steadystate solutions," Journal of Differential Equations, vol. 39, no. 2, pp. 269-290, 1981.

[7] S.-H. Wang and D.-M. Long, "An exact multiplicity theorem involving concave-convex nonlinearities and its application to stationary solutions of a singular diffusion problem," Nonlinear Analysis. Theory, Methods \& Applications A, vol. 44, pp. 469486, 2001

[8] L. Q. Zhang, "Uniqueness of positive solutions of $\Delta u+u+u^{p}=0$ in a ball," Communications in Partial Differential Equations, vol. 17, no. 7-8, pp. 1141-1164, 1992.

[9] Z. Liu, "Exact number of solutions of a class of two-point boundary value problems involving concave and convex nonlinearities," Nonlinear Analysis. Theory, Methods \& Applications A, vol. 46, pp. 181-197, 2001.

[10] Z. Liu and X. Zhang, "A class of two-point boundary value problems," Journal of Mathematical Analysis and Applications, vol. 254, no. 2, pp. 599-617, 2001.

[11] J. Cheng, "Exact number of positive solutions for a class of semipositone problems," Journal of Mathematical Analysis and Applications, vol. 280, no. 2, pp. 197-208, 2003. 


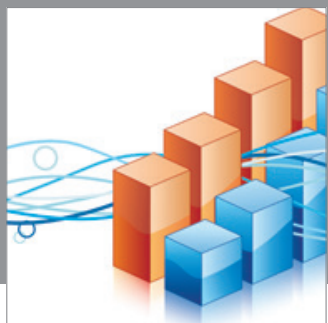

Advances in

Operations Research

mansans

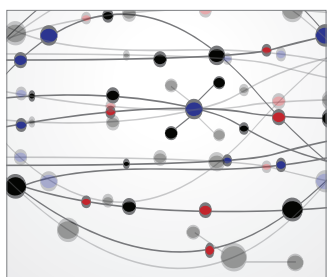

The Scientific World Journal
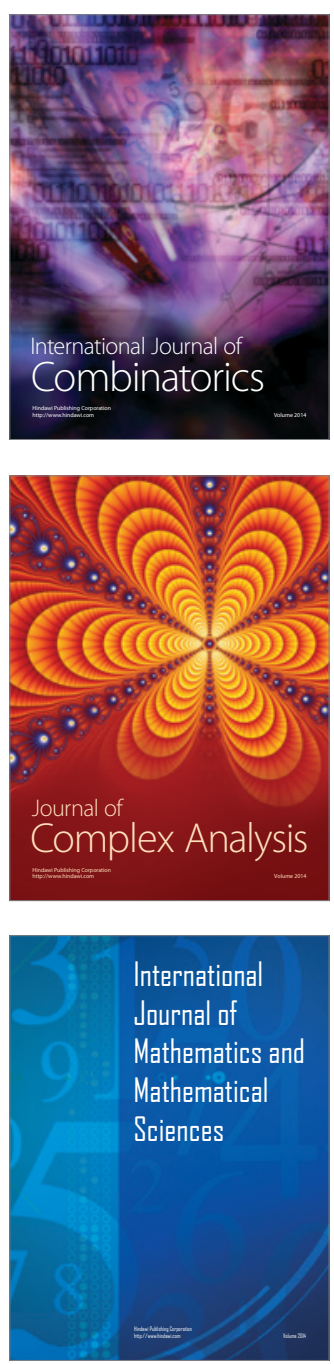
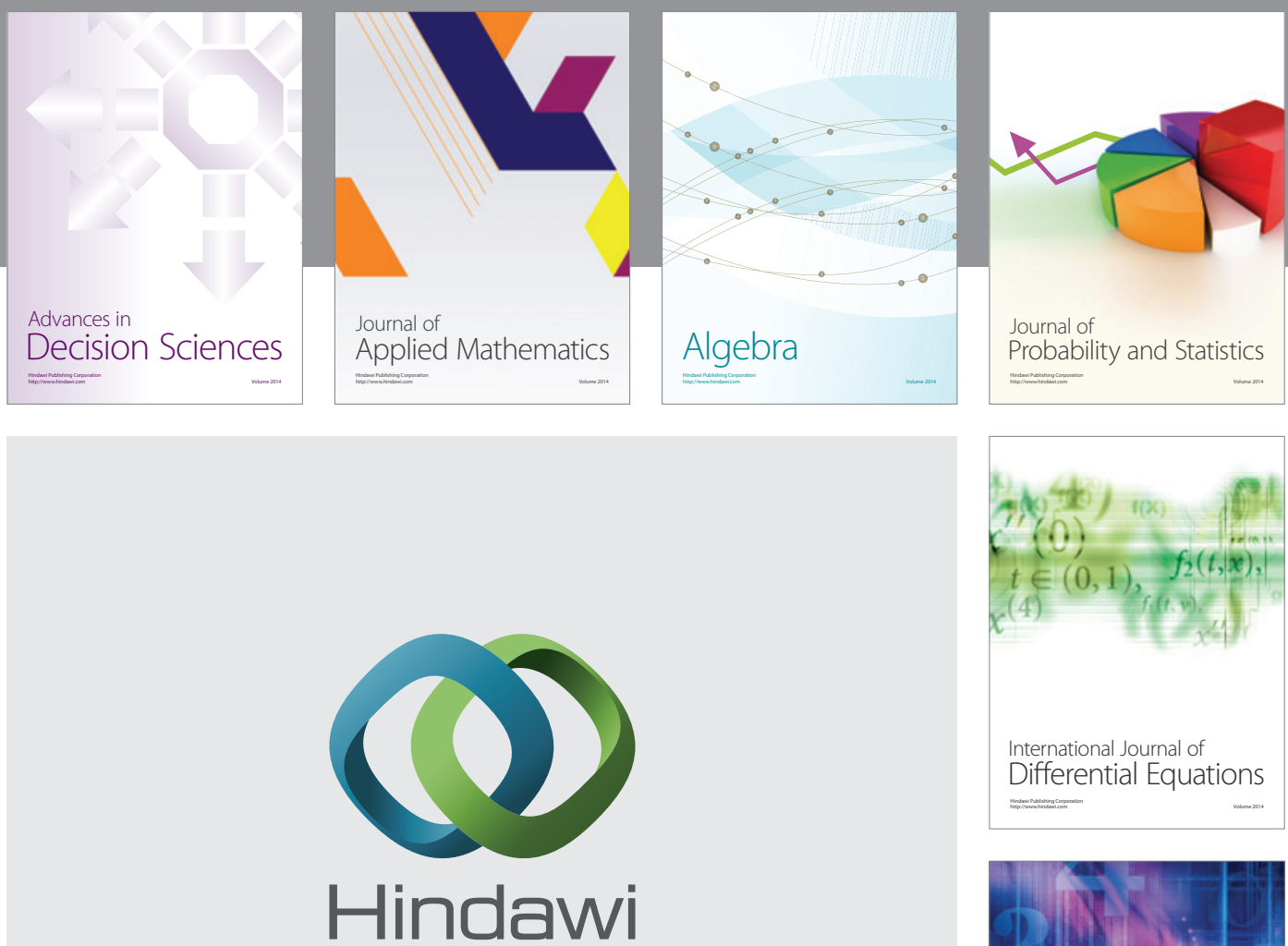

Submit your manuscripts at http://www.hindawi.com
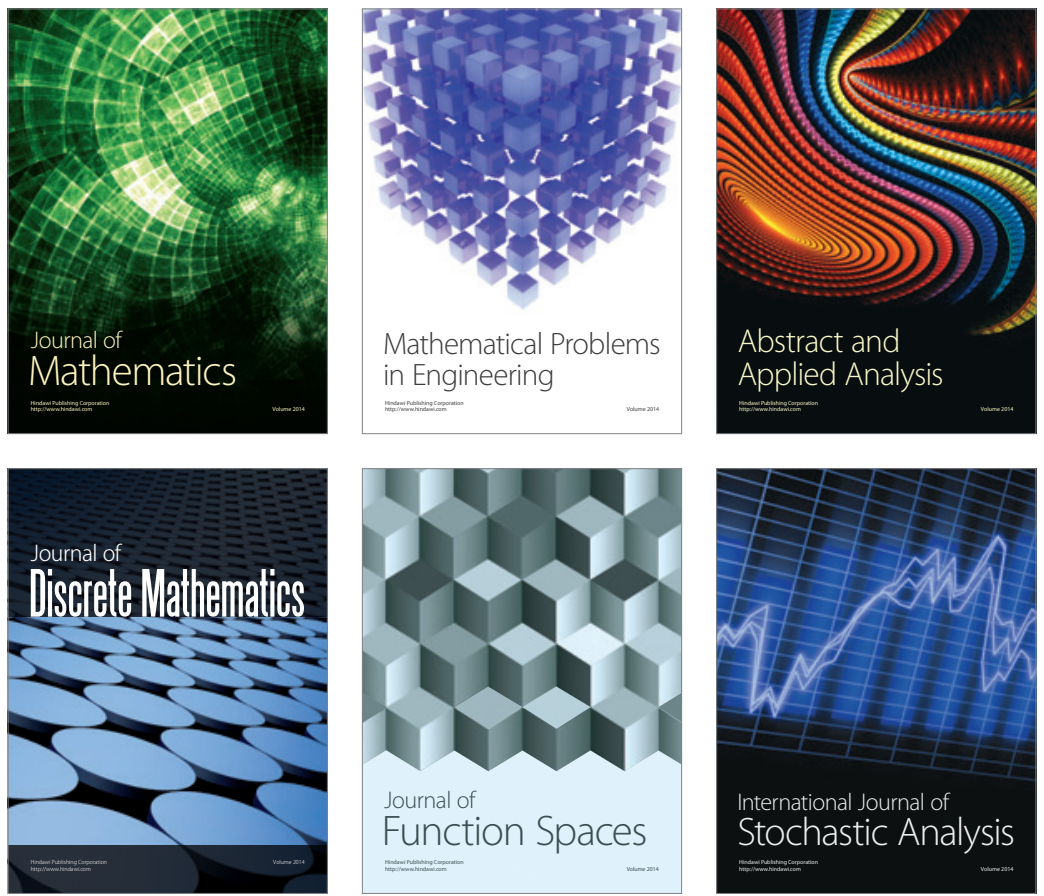

Journal of

Function Spaces

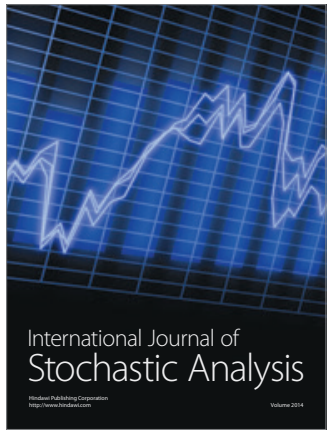

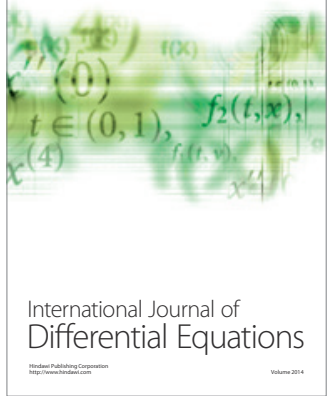
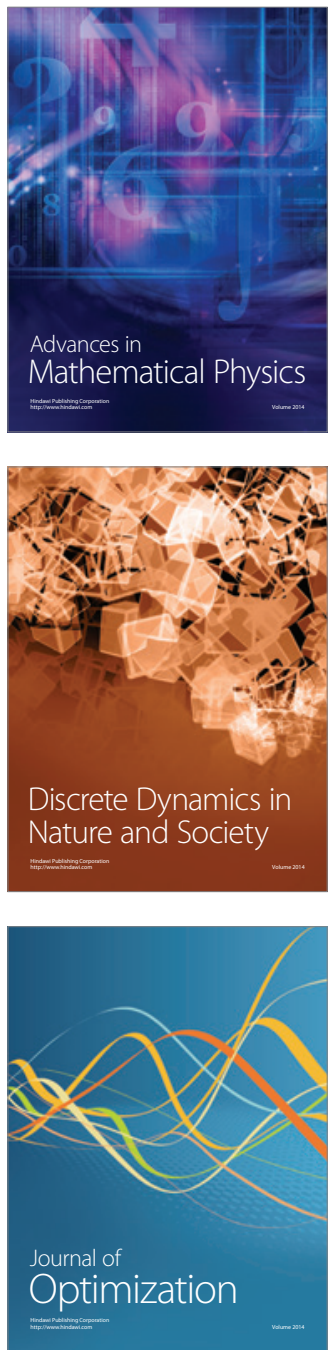